\title{
Sugeno Inference Perturbation Analysis for Electric Aerial Vehicles
}

\author{
John T. Economou and Kevin Knowles \\ Intelligent Propulsion and Emissions Laboratory \\ Aeromechanical Systems Group \\ Cranfield University, Defence Academy of the United Kingdom
}

UK

\section{Introduction}

The Chapter is focusing in the area of the Electrically-powered Unmanned Aerial Vehicles (UAVs) in association to bounded sensor noise. The increased requirements for airworthiness and safety of such vehicles have resulted in the requirement of improving the analytical methods for subsystem level mathematical modelling, such as for example the electrical propulsion system. The Takagi-Sugeno fuzzy inference has been formulated in the context of bounded multi-sensor errors for a range of error classes. The modelled system is an electrical propulsion system together with the associated sensor boundaries in relation to a typical UAV operation.

Unmanned aerial vehicles have been used in various operational conditions where other vehicles fail to operate. UAVs have been used to inspect hazardous areas such as flooded areas, earthquake areas, and generally areas that may have a high risk of radioactive contaminants. The immediate result of the effective use of UAVs is reducing the risk of endangering human lives while still capable of operating safely and efficiently. This chapter addresses the issue of sensor operational boundaries and the UAV's electrical thruster parametric variation due to altitude variations. UAVs normally operate over a range of altitudes Kladis et al. (2010) depending on their operational role. Hence, these can be exposed to a range of temperature conditions which can affect their normal operation. This chapter addresses this specific consideration which can have airworthiness implications, and focuses on the description of the electrical permanent magnet direct current thrusters in the context of UAVs and operations .

The UAV's propulsion options can vary depending on the user and operational requirements, however the focal point for the work described in this chapter is for an electrical thruster system. Such systems being supplied from a fuel cell are described in more detail in Karunarathne et al. (2007). In particular, the work in Karunarathne et al. (2007), describes for a given example UAV operation the effectiveness of the electric propulsion option together with the importance of a sophisticated power management system utilising intelligent based methods. In Miller (2004), the propulsion system options are described within the context of power and energy and thus assist towards the importance of electro-mechanical systems for propulsion. In Ehsani et al. (2005), the authors 
contribute towards a structured approach towards the theory of propulsion systems and the general design considerations surrounding these systems. Both resources together with this chapter will enhance the readers' awareness towards the power and energy design considerations in relation to sensing and the boundaries that these systems have when related to UAV operations.

In particular in this chapter, the electrical thruster is modelled as an ordinary differential equation which can operate in either motoring or generator mode depending on the operational shaft angular velocity and the motor torque. The theoretical parts present the Sugeno fuzzy inference in association to the fuzzy-hybrid concept developed by Economou \& Colyer (2005). The latter is demonstrated from the simulated behaviour of the PMDC thruster. Part of the electrical thruster, based on Economou \& Colyer (2005) can be presented in an ordinary differential equation representation while when the UAV altitude is included then the model exerts partially a Sugeno type fuzzy behaviour. The collection of these behaviours is shown in this chapter.

In effect part of the thruster is modelled utilising physical system modelling methods while the remaining part of the system is modelled using an intelligent based method (fuzzy logic). Overall the thruster is a fuzzy-hybrid system as per Economou \& Colyer (2005). In particular, the fuzzy inference system utilised in this chapter is a Sugeno system Sugeno (1999).

Furthermore when a system is realised in practice it is also highly likely to contain some deviations from its nominal measurements Economou et al. (2007). The sensors are expected due to operational temperature variations for example to incorporate an error deviating from the nominal value. For the UAV electrical thruster the consequence is that the thruster angular velocity will tend to deviate from the expected nominal value and this could lead to loss of aerodynamic propeller thrust and can therefore lead to airworthiness and safety implications. This chapter clearly shows that the thruster's angular velocity can vary from its nominal (expected value), when the additional effects of sensor error boundaries and temperature variation (due to altitude), are both included in the mathematical modelling. The resulting analysis is demonstrated for a given operational UAV scenario, indicating that the percentage errors exceeded the value of $20 \%$ over the nominal value for the armature thruster's resistance.

\section{Analysis}

\subsection{Sugeno output perturbation}

Fuzzy logic is a methodology which results in representing a system or controlling a system using If-Then rules. For the purpose of this Chapter a Sugeno type inference is utilised as a modelling tool. The system $n$-th rule can be represented as follows:

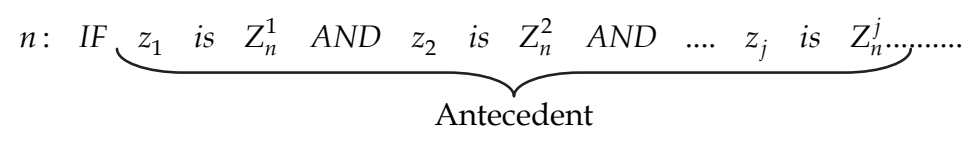

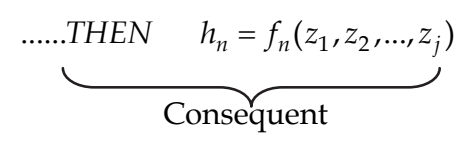


The membership functions represent the belonging of the sampled variable at a specific time instant $t=\tau$ to the specific membership function $Z_{n}^{j}$ for rule $(n)$ and sensor $(j)$. The corresponding (j-th) membership functions which are not the left and right edge membership functions are Gaussian type functions (1):

$$
Z_{n}^{j}\left(z_{j}\right)=e^{-\frac{\left(z_{j}-c_{j}\right)^{2}}{d_{j}}}
$$

(1) is valid for $n=2,3, \ldots, n_{\max }-1$ i.e. the membership functions representing the centred membership functions. The left edge $(n=1)$ and right edge $\left(n=n_{\max }\right)$ are sigmoid type of membership functions. These are given from the following expressions $(2 a)$ and $(2 b)$ :

$$
Z_{n}^{j}=\left\{\begin{aligned}
Z_{1}^{j} & =\frac{1}{1+e^{z_{j}}} \\
Z_{n_{\max }}^{j} & =\frac{1}{1+\eta e^{-z_{j}}}
\end{aligned}\right.
$$

The graphical illustration of the membership functions is shown next in Figure 1 for the $j$-th sensor.

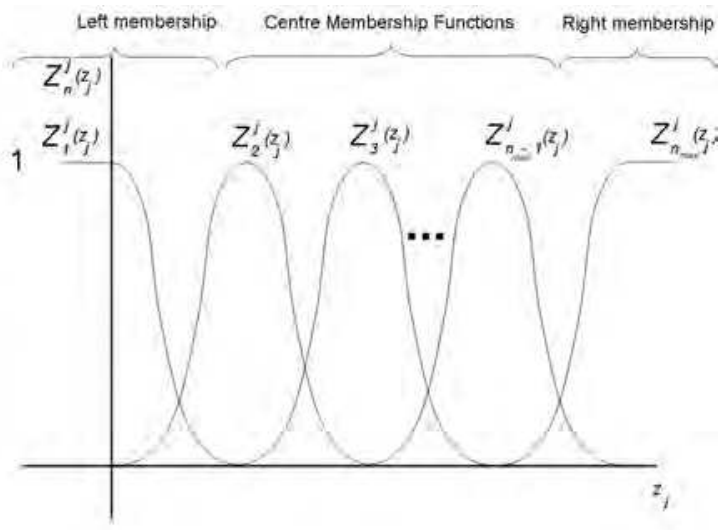

Fig. 1. Generalised Membership Functions for the $j$-th sensor

The polynomial for each Sugeno rule is given from the following expression (3):

$$
\forall n: h_{n}:=b_{n}+\sum_{j=1}^{j_{\max }} \varsigma_{j n} z_{j}
$$

Based on the general Sugeno rule description the resulting defuzzyfied output is given from the following expanded equation (4) for time $t=\tau$ which represents the nominal system response: 


$$
H^{*}(\tau)=\frac{\gamma_{1} h_{1}+\gamma_{2} h_{2}+\ldots+\gamma_{n_{\max }} h_{n_{\max }}}{\gamma_{1}+\gamma_{2}+\ldots+\gamma_{n_{\max }}}=\frac{\sum_{n}^{n_{\max }} \gamma_{n} h_{n}}{\sum_{n}^{n_{\max }} \gamma_{n}}
$$

The antecedent "AND" operator results into the following expressions (5):

$$
\begin{aligned}
& \gamma_{1}=Z_{1}^{1}\left(z_{1}\right) \cdot Z_{1}^{2}\left(z_{2}\right) \cdot Z_{1}^{3}\left(z_{3}\right) \ldots \cdot Z_{1}^{j_{\max }}\left(z_{j_{\max }}\right) \\
& \gamma_{2}=Z_{1}^{1}\left(z_{1}\right) \cdot Z_{1}^{2}\left(z_{2}\right) \cdot Z_{1}^{3}\left(z_{3}\right) \ldots \cdot Z_{2}^{j_{\max }}\left(z_{j_{\max }}\right) \\
& \gamma_{3}=Z_{1}^{1}\left(z_{1}\right) \cdot Z_{1}^{2}\left(z_{2}\right) \cdot Z_{1}^{3}\left(z_{3}\right) \ldots \cdot Z_{3}^{j_{\max }}\left(z_{j_{\max }}\right) \\
& \gamma_{4}=Z_{1}^{1}\left(z_{1}\right) \cdot Z_{2}^{2}\left(z_{2}\right) \cdot Z_{1}^{3}\left(z_{3}\right) \ldots \cdot Z_{1}^{j_{\max }}\left(z_{j_{\max }}\right) \\
& \gamma_{5}=Z_{1}^{1}\left(z_{1}\right) \cdot Z_{2}^{2}\left(z_{2}\right) \cdot Z_{1}^{3}\left(z_{3}\right) \ldots \cdot Z_{2}^{j_{\max }}\left(z_{j_{\max }}\right) \\
& \gamma_{6}=Z_{1}^{1}\left(z_{1}\right) \cdot Z_{2}^{2}\left(z_{2}\right) \cdot Z_{1}^{3}\left(z_{3}\right) \ldots \cdot Z_{3}^{j_{\max }}\left(z_{j_{\max }}\right) \\
& \vdots \\
& \vdots \\
& \gamma_{n_{\max }}=Z_{n_{\max }}^{1}\left(z_{1}\right) \cdot Z_{n_{\max }}^{2}\left(z_{2}\right) \cdot Z_{n_{\max }}^{3}\left(z_{3}\right) \ldots \cdot Z_{n_{\max }}^{j_{\max }}\left(z_{j_{\max }}\right)
\end{aligned}
$$

From (5) it can be deduced that if the "left" edge triggers only uniformly for all rules $\left(n_{\max }\right)$, then the following equality (6) holds:

$$
\gamma_{1}=Z_{1}^{1}\left(z_{1}\right) \cdot Z_{1}^{2}\left(z_{2}\right) \cdot Z_{1}^{3}\left(z_{3}\right) \ldots . \cdot Z_{1}^{j_{\max }}\left(z_{j_{\max }}\right)
$$

It can also be deduced that if a "right" edge trigger only triggers then the following equality holds (7):

$$
\gamma_{n_{\max }}=Z_{n_{\max }}^{1}\left(z_{1}\right) \cdot Z_{n_{\max }}^{2}\left(z_{2}\right) \cdot Z_{n_{\max }}^{3}\left(z_{3}\right) \ldots \cdot Z_{n_{\max }}^{j_{\max }}\left(z_{j_{\max }}\right)
$$

For all other remaining conditions the following "centred" rules can trigger as shown from the set of equations in (8):

$$
\begin{aligned}
& \gamma_{2}=Z_{1}^{1}\left(z_{1}\right) \cdot Z_{1}^{2}\left(z_{2}\right) \cdot Z_{1}^{3}\left(z_{3}\right) \ldots \cdot Z_{2}^{j_{\max }}\left(z_{j_{\max }}\right) \\
& \gamma_{3}=Z_{1}^{1}\left(z_{1}\right) \cdot Z_{1}^{2}\left(z_{2}\right) \cdot Z_{1}^{3}\left(z_{3}\right) \ldots \cdot Z_{3}^{j_{\max }}\left(z_{j_{\max }}\right) \\
& \gamma_{4}=Z_{1}^{1}\left(z_{1}\right) \cdot Z_{2}^{2}\left(z_{2}\right) \cdot Z_{1}^{3}\left(z_{3}\right) \ldots \cdot Z_{1}^{j_{\max }}\left(z_{j_{\max }}\right) \\
& \gamma_{5}=Z_{1}^{1}\left(z_{1}\right) \cdot Z_{2}^{2}\left(z_{2}\right) \cdot Z_{1}^{3}\left(z_{3}\right) \ldots \cdot Z_{2}^{j_{\max }}\left(z_{j_{\max }}\right) \\
& \gamma_{6}=Z_{1}^{1}\left(z_{1}\right) \cdot Z_{2}^{2}\left(z_{2}\right) \cdot Z_{1}^{3}\left(z_{3}\right) \ldots \cdot Z_{3}^{j_{\max }}\left(z_{j_{\max }}\right) \\
& \vdots \\
& \gamma_{n_{\max }-1}=Z_{n_{\max }-1}^{1}\left(z_{1}\right) \cdot Z_{n_{\max }-1}^{2}\left(z_{2}\right) \cdot Z_{n_{\max }-1}^{3}\left(z_{3}\right) \ldots \cdot Z_{n_{\max }-1}^{j_{\max }}\left(z_{j_{\max }}\right)
\end{aligned}
$$




\subsection{Sugeno output perturbation models}

Based on the research work in Economou \& Colyer (2005) it can be deduced that the preferred architecture of a fuzzy-hybrid is the following equation (9):

$$
y=f_{1}(\underline{p}) u_{1}+f_{2}(\underline{p}) g\left(u_{2}\right) \mathrm{H}^{*}+f_{3}(\underline{p})
$$

Where $f_{1}(p), f_{2}(p), f_{3}(p)$ are the parametric functions with respect to a vector $p . u_{1}, u_{2}$ are the fuzzy-hybrid system inputs. And $g\left(u_{2}\right)$ is a function of the input $u_{2}$. The mathematical expression (9) will be associated to the electric propulsion equation. The $\left(\mathrm{H}^{*}\right)$ term represents the fuzzy Sugeno non-singleton type system which will associate to sensor perturbations $\delta \beta_{n}=\varepsilon_{n}$ and thus observe how key variables can potentially drift from their expected nominal value for given conditions. Hence, by incorporating the work in Economou et al. (2007), the Singleton type inference is provided from the following equation:

$$
\begin{gathered}
H_{+}^{*}=\frac{\sum_{n=1}^{n_{\max }} \gamma_{n} h_{n}+\sum_{n=1}^{n_{\max }}\left(\gamma_{n} \delta \beta_{n}\right)}{\sum_{n=1}^{n_{\max }} \gamma_{n}} \\
H_{-}^{*}=\frac{\sum_{n=1}^{n_{\max }} \gamma_{n} h_{n}-\sum_{n=1}^{n_{\max }}\left(\gamma_{n} \delta \beta_{n}\right)}{\sum_{n=1}^{n_{\max }} \gamma_{n}}
\end{gathered}
$$

Which assumes that (11) is true for (10a) and (10b).

$$
\sum_{j=1}^{j_{\max }} \varsigma_{j n} z_{j} \neq 0
$$

Where the term $\delta \beta_{n}$ is the perturbation for the $\mathrm{n}$-th rule for the given antecedent conditions.

\subsection{Static error bound models}

\subsubsection{Class of Static Isotropic Error Bounds (SIEB)}

For this class of errors we have a set with lower and upper bounds for each sensor $(j)$, $S_{1}: \varepsilon_{j} \in\left[\underline{\varepsilon}_{j}, \bar{\varepsilon}_{j}\right]$. These errors are valid for the entire observation interval $t \in\left[0, t_{f}\right]$. For this class of errors it is possible that the errors for each sensor $(j)$ are equal. Hence we could have the special case that for the sets,

$$
\begin{aligned}
& S_{1}: \varepsilon_{1} \in\left[\underline{\varepsilon}_{1},-\bar{\varepsilon}_{1}\right]=\left[-\bar{\varepsilon}_{1},-\bar{\varepsilon}_{1}\right], \varepsilon_{2} \in\left[\underline{\varepsilon}_{2},-\bar{\varepsilon}_{2}\right]=\left[-\overline{\varepsilon_{2}},-\varepsilon_{2}\right] \ldots . \varepsilon_{j} \in\left[\underline{\varepsilon}_{j}, \bar{\varepsilon}_{j}\right]=\left[-\bar{\varepsilon}_{j}, \bar{\varepsilon}_{j}\right], \\
& \underline{\varepsilon}_{1}=\underline{\varepsilon}_{2}=\ldots .=\underline{\varepsilon}_{j}, \bar{\varepsilon}_{1}=\bar{\varepsilon}_{2}=\ldots \bar{\varepsilon}_{j},
\end{aligned}
$$

Although it is more often the case that the following condition will be true: $\underline{\varepsilon}_{1} \neq \underline{\varepsilon}_{2} \neq \ldots \neq \underline{\varepsilon}_{j}, \bar{\varepsilon}_{1} \neq \bar{\varepsilon}_{2} \neq \ldots \bar{\varepsilon}_{j}$. 


\subsubsection{Class of Static Anisotropic Error Bounds (SAEB)}

In the case the upper and lower bound errors are considered to be anisotropic which therefore result in the following condition:

$$
S_{2}: \varepsilon_{1} \in\left[\underline{\varepsilon}_{1}, \bar{\varepsilon}_{1}\right], \varepsilon_{2} \in\left[\underline{\varepsilon}_{2}, \overline{\varepsilon_{2}}\right] \ldots . \varepsilon_{j} \in\left[\underline{\varepsilon}_{j}, \bar{\varepsilon}_{j}\right], \underline{\varepsilon}_{1} \neq-\bar{\varepsilon}_{1}, \underline{\varepsilon}_{2} \neq-\bar{\varepsilon}_{2}, \ldots ., \underline{\varepsilon}_{j} \neq-\bar{\varepsilon}_{j}
$$

\subsubsection{Class of Static Clustered Isotropic Error Bounds (SCIEB)}

For this particular class of systems identical classes of sensors can used in order to acquire experimental data. For these cases the numbering order of the sensors will result in a unique system representation. Hence the following figure can be used in order to refer to a selection of choices,

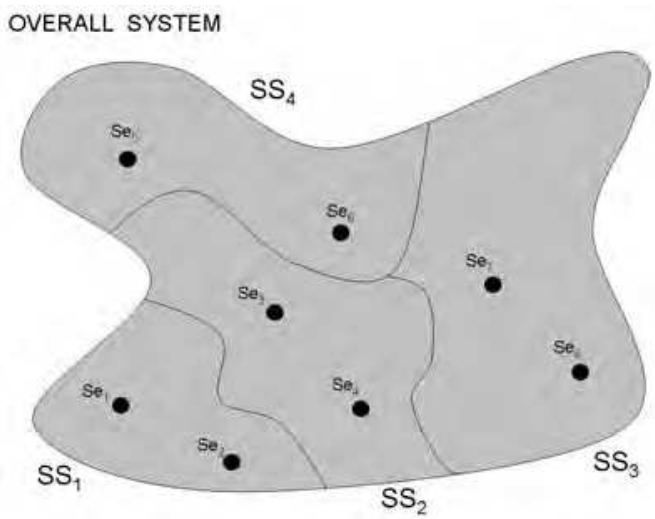

Fig. 2. SCIEB representation for a Generalised System with 8 sensors (Se) and 4 clusters (SS).

For this system the following expression exists:

$$
\begin{aligned}
& S S_{1}: \varepsilon_{1} \in\left[\underline{\varepsilon}_{1}, \bar{\varepsilon}_{1}\right], \varepsilon_{2} \in\left[\underline{\varepsilon}_{2}, \bar{\varepsilon}_{2}\right], \underline{\varepsilon}_{1}=-\bar{\varepsilon}_{1}=\underline{\varepsilon}_{2}=-\bar{\varepsilon}_{2} \\
& S S_{2}: \varepsilon_{3} \in\left[\underline{\varepsilon}_{3}, \bar{\varepsilon}\right], \varepsilon_{4} \in\left[\underline{\varepsilon}_{4}, \bar{\varepsilon}_{4}\right], \underline{\varepsilon}_{3}=-\bar{\varepsilon}_{3}=\underline{\varepsilon}_{4}=-\bar{\varepsilon} 4 \\
& S S_{3}: \varepsilon_{7} \in\left[\underline{\varepsilon}_{7},-\bar{\varepsilon}_{7}\right], \varepsilon_{8} \in\left[\underline{\varepsilon}_{8},-\bar{\varepsilon}_{8}\right], \underline{\varepsilon}_{7}=-\bar{\varepsilon}_{7}=\underline{\varepsilon}_{8}=-\bar{\varepsilon}_{8} \\
& S S_{5}: \varepsilon_{5} \in\left[\underline{\varepsilon}_{5}, \bar{\varepsilon} 5\right], \varepsilon_{6} \in\left[\underline{\varepsilon}_{6},-\bar{\varepsilon} 6\right], \underline{\varepsilon}_{5}=-\bar{\varepsilon}_{5}=\underline{\varepsilon}_{6}=-\bar{\varepsilon} 6
\end{aligned}
$$

Figure 2 for the same system, sensors and clusters is not unique because it is based on the ordering of the subsystems and the ordering of the individual sensors.

\subsection{Dynamic error bound models}

The dynamic error bounds are time based and therefore represent the variation in a polynomial form and can be similarly divided into three main categories similar to the static case but with the error bounds being represented in a polynomial form. These are divided into the Class of dynamic isotropic error bounds (DIEB), Class of dynamic anisotropic error bounds (DAEB), Class of dynamic clustered isotropic error bounds (DCIEB). The results shown for the UAV application are based on the SIEB type of errors for illustration purposes. 


\subsection{Relation of sugeno perturbation and error type classification}

For the SIEB type of perturbation equations (10a) and (10b) hold, while for the case of SAEB the perturbations are unequal for each sensor and therefore the following expression holds:

$$
\begin{gathered}
\mathrm{Z}_{+}^{*}=\frac{\sum_{n=1}^{n_{\max }} \gamma_{n} h_{n}+\sum_{n=1}^{n_{\max }}\left(\gamma_{n} \delta \beta_{n}^{+}\right)}{\sum_{n=1}^{n_{\max }} \gamma_{n}} \\
\mathrm{Z}_{-}^{*}=\frac{\sum_{n=1}^{n_{\max }} \gamma_{n} h_{n}-\sum_{n=1}^{n_{\max }}\left(\gamma_{n} \delta \beta_{n}^{-}\right)}{\sum_{n=1}^{n_{\max }} \gamma_{n}} \\
\delta \beta_{n}^{+} \neq \delta \beta_{n}^{-}
\end{gathered}
$$

Where $\delta \beta_{n}^{+} \neq \delta \beta_{n}^{-}$corresponds to the asymmetry of the perturbations in the consequent fuzzy component. Subject to the constraint $(12 \mathrm{~d})$ :

$$
\sum_{j=1}^{j_{\max }} \varsigma_{j n} z_{j} \neq 0
$$

\subsection{Application: Electric aerial vehicle propulsion system \\ 2.6.1 System description}

The system is an unmanned aerial vehicle electrical propulsion permanent magnet system linked via a gearbox to the propeller. It is assumed that suitable power electronics/controls and energy sources are in place for supplying the electrical thrusters. The aerial vehicle is capable of flying over a range of altitudes and therefore the thrusters and propeller are capable of meeting a range of angular velocity and load torque demands.

\subsubsection{Mathematical problem modelling}

The electric machine (permanent magnet d.c.) is modelled as a dual mode ordinary differential equation representing using fuzzy switching the two operational modes.

Case 1: Motoring mode (Torque, speed quadrants 1,3):

$$
V_{a}(t)=K_{a} \omega(t)+R_{a} i_{a}(t)+L \frac{d i_{a}(t)}{d t}
$$

Case 2: Generator mode (Torque, speed quadrants 2,4):

$$
E_{a}(t)=V_{a}(t)+R_{a} i_{a}(t)+L \frac{d i_{a}(t)}{d t}
$$

For case 1, an applied external voltage is required in order to provide rotor motion while also the motor provided torque is sufficient to drive at any given time the applied load and 
mathematically presented in (13a). For case 2, the expression is shown in (13b) while the rotor is rotating due to an external mechanical force (generation) as long as the back emf voltage is higher than $V_{a}$ then generation occurs (it is assumed that the power electronics will satisfactory re-root the power back into a rechargeable battery source and therefore store energy). For both modes the following equations are valid. The motor back emf is provided from $(13 c)$ :

$$
E_{a}=K_{a} \omega(t)
$$

The rotor angular velocity is given from $(13 \mathrm{~d})$ :

$$
\frac{d \theta(t)}{d t}=\omega(t)
$$

The motor shaft torque is given from (14):

$$
T_{m}(t)=i_{a}(t) K_{T}
$$

The motor supplied torque is linked to the mechanical system load as shown next (15):

$$
T_{m}(t)=\left(J_{a}+J_{L}\left(\frac{N_{1}}{N_{2}}\right)^{2}\right) \frac{d^{2} \theta}{d t^{2}}+\left(B_{a}+B_{L}\left(\frac{N_{1}}{N_{2}}\right)^{2}\right) \frac{d \theta}{d t}
$$

Revisiting the equations from case 1 and case 2 can both be generalised and result in equation (16):

$$
V_{a}(t)=K_{a} \omega(t)+\operatorname{sign}\left(P_{m}\right) \cdot R_{a} i_{a}(t)+\operatorname{sign}\left(P_{m}\right) \cdot L \frac{d i_{a}(t)}{d t}
$$

The "sign" function is provided as shown next in (17):

$$
\operatorname{sign}\left(P_{m}\right)=\left\{\begin{array}{cc}
+1 & P_{m}>0 \\
0 & P_{m}=0 \\
-1 & P_{m}<0
\end{array}\right.
$$

Alternatively the sign function can also be approximated to equation (18):

$$
\operatorname{sign}\left(P_{m}(t)\right) \approx \frac{P_{m}(t)}{\sqrt{\left(P(t)_{m}^{2}+\rho^{2}\right)}}=\psi\left(P_{m}(t), \rho\right), \rho \in \mathfrak{R}^{+}
$$

The variable $P_{m}$ is the mechanical motor power. Modes 1 and 2 from (16) and equation (18) will result in the following expression (19).

$$
V_{a}(t)=K_{a} \omega(t)+\frac{P_{m}(t)}{\sqrt{P(t)_{m}^{2}+\rho^{2}}} R_{a} i_{a}(t)+\frac{P_{m}(t)}{\sqrt{P(t)_{m}^{2}+\rho^{2}}} L \frac{d i_{a}(t)}{d t}
$$

Allowing an armature resistance variation with reference to the aerial vehicle altitude $(h)$ and environmental conditions such as air moisture parameter $(\zeta)$ given from equation $(20)$ : 


$$
R_{a}=R_{r e f}\left[1+\alpha_{c}\left(T-T_{r e f}\right)\right]=f(\zeta, \xi)
$$

Hence by combining the results from (19) and (20) the following expression is obtained (21):

$$
V_{a}(t)=K_{a} \omega(t)+\frac{P_{m}(t)}{\sqrt{P(t)_{m}^{2}+\rho^{2}}} i_{a}(t) f(\zeta, \xi)+\frac{P_{m}(t)}{\sqrt{P(t)^{2}+\rho^{2}}} L \frac{d i_{a}(t)}{d t}
$$

This can be simplified to equation (22):

$$
V_{a}(t)=\underbrace{K_{a} \omega(t)}_{\text {Term } 1}+\underbrace{\psi\left(P_{m}(t), \rho\right) i_{a}(t) f(\zeta, \xi)}_{\text {Term } 2}+\underbrace{\psi\left(P_{m}(t), \rho\right) L \frac{d i_{a}(t)}{d t}}_{\text {Term } 3}
$$

With reference to equation (22), term 1 represents the mechanical equivalent voltage which causes the aircraft propeller to rotate. Term 2, corresponds to the motor windings copper voltage loss which is temperature-sensitive, due to a change of aircraft altitude and air moisture for example. The third term relates to the propeller motor thrust equivalent voltage. When a change of thrust is required for the same shaft angular velocity, then the current will vary with time and therefore the inductive element will become active. The ordinary differential equation with respect to the thruster armature current is given from (23):

$$
\frac{d i_{a}(t)}{d t}=-\frac{1}{\psi\left(P_{m}(t), \rho\right) L}\left(K_{a} \omega(t)-\psi\left(P_{m}(t), \rho\right) i_{a}(t) f(\zeta, \xi)\right)+\frac{1}{\psi\left(P_{m}(t), \rho\right) L} V_{a}(t)
$$

Equation (23) as time $t \rightarrow 0$ can be simplified to the following expression (24a):

$$
V_{a}(t)=K_{a} \omega(t)+\psi\left(P_{m}(t), \rho\right) f(\zeta, \xi) i_{a}(t)
$$

Equation (24) can be rearranged to obtain the EPS angular velocity (24b).

$$
\omega(t)=-\psi\left(P_{m}(t), \rho\right) f(\zeta, \xi) \frac{1}{K_{a}} i_{a}(t)+\frac{1}{K_{a}} V_{a}(t)
$$

When (24b) is compared to the fuzzy-hybrid topology shown in (9) the following equalities are valid:

$$
\begin{aligned}
& y=\omega(t) \\
& f_{1}(\underline{p})=\frac{1}{K_{a}} \\
& u_{1}=V_{a}(t) \\
& f_{2}(\underline{p})=-\frac{\psi\left(P_{m}(t), \rho\right)}{K_{a}} \\
& f_{3}(\underline{p})=0 \\
& g\left(u_{2}\right)=i_{a}(t) \\
& H^{*}=f(\zeta, \xi)
\end{aligned}
$$




\subsubsection{Electrical propulsion power consumers}

The electrical propulsion system has several power consumers. In order to illustrate these, the power flow equation needs to be considered first which relates the input thruster power to the power consumers.

The power inserted to the electrical actuator $P_{a}(t)$ is provided from the following expression (25):

$$
P_{a}(t)=\underbrace{K_{a} \omega(t) i_{a}(t)}_{\text {Term1 }}+\underbrace{\psi\left(P_{m}(t), \rho\right) i_{a}^{2}(t) f(\zeta, \xi)}_{\text {Term2 }}+\underbrace{\psi\left(P_{m}(t), \rho\right) L \frac{d i_{a}(t)}{d t} i_{a}(t)}_{\text {Term3 }}
$$

In equation (25) term 3 is normally non-zero when there is a change in thrust and therefore armature current and can be neglected for quasi-static conditions. Term 2 represents the conductive armature resistance losses while the useful power is the mechanical power shown as term 1 . Normally, the following inequality (26) is desired:

$$
K_{a} \omega(t) i_{a}(t)>>\psi\left(P_{m}(t), \rho\right) i_{a}^{2}(t) f(\zeta, \xi)+\psi\left(P_{m}(t), \rho\right) L \frac{d i_{a}(t)}{d t} i_{a}(t)
$$

However in practice efficiencies can vary depending on the angular velocity and loading over a wide operational envelope. For a UAV application the expected efficiencies are typically very high due to the near to optimum angular velocity operation.

\subsection{Sensor class and simulation demonstration of implications to the electric application}

For the Unmanned Aerial Vehicle (UAV) application our objective is to investigate the effects that the user implicitly incurs to the UAV. In particular when the UAV operator, due to mission requirements, selects to change altitude in the range of $0-6 \mathrm{~km}$, for example, then the ambient temperature conditions can cause the temperature to drop several degrees $(\mathrm{K})$ per $1000 \mathrm{~m}$ increase in altitude $(7 \mathrm{~K} / \mathrm{km})$ for given moisture conditions.

Consequently, the electrical propulsion system will experience a temperature drop which results in variation of the coil armature resistance. Therefore, the angular velocity of the propulsion will be affected thus resulting in further changes to the UAV propeller thrust.

The purpose of this analysis is to demonstrate the effects of these variations and error tolerance in the temperature sensing and show how these can affect UAV performance via the loss of thrust. Figure 3 illustrates this:

The exogenous altitude variations represent the source of altitude and air moisture which both affect the ambient temperature and therefore both can affect the UAV operation and deviate this from its nominal (or expected) behaviour due to variations in the Electrical Propulsion System (EPS).

Although Figure 3, shows four interconnections in essence these are repetitive. After the first sequence from stage 1 to 4 has lapsed then the operator does receive visual feedback and therefore reacts in order to compensate according to the mission plan. Therefore, the effects of the EPS parametric variation and the effects of the perturbation for the temperature sensing boundaries will be investigated, as shown in Figure 4. 


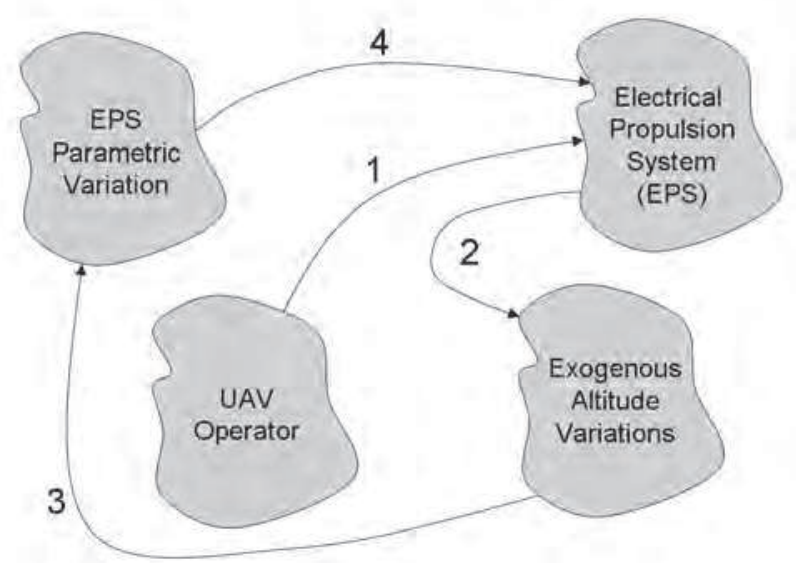

Fig. 3. UAV operator and EPS performance for varying altitudes

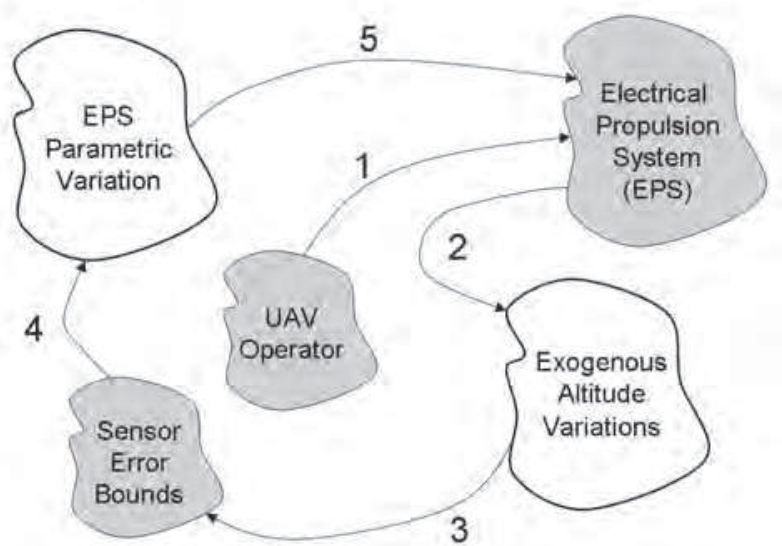

Fig. 4. EPS Parametric Variation and Exogenous Altitude Variations

The proposed simulation block diagram for the UAV which incorporates a perturbation and the fuzzy-hybrid model for the UAV thrusters is presented as shown next in Figure 5:

Figure 6 shows the system's operational requirements for a near to sea level UAV altitude thus having overall a constant armature resistance. Figure 6. shows a similar block diagram which includes the UAV Altitude Profile, UAV "dry/moist" profile which provide an estimate for the perturbed temperature via a Sugeno-type Fuzzy Inference System (FIS). The armature resistance variation with temperature and the electrical thrusters are based on physical system modelling. The fuzzy Sugeno system produces a nominal armature resistance variation which is related to the UAV altitude and air moisture conditions. Lastly figure 8 shows the Sugeno FIS which produces the perturbed armature resistance values for the electrical thruster. Two inputs, the UAV armature voltage and UAV current profile are 
driving the electrical thruster for the given airframe and associated aerodynamics. Meanwhile the thruster's armature resistance will vary significantly depending on the UAV altitude. Furthermore, the thruster's angular velocity variation can be observed for given system demands and compared to the nominal and the expected (perturbation) values obtained in the later figures clearly showing the key variations.

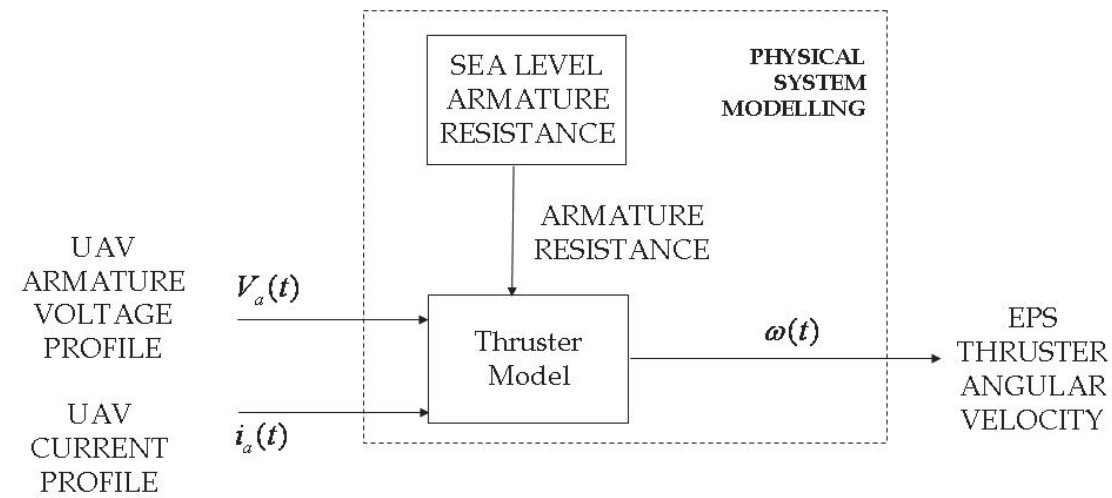

Fig. 6. UAV EPS Model near sea level altitude

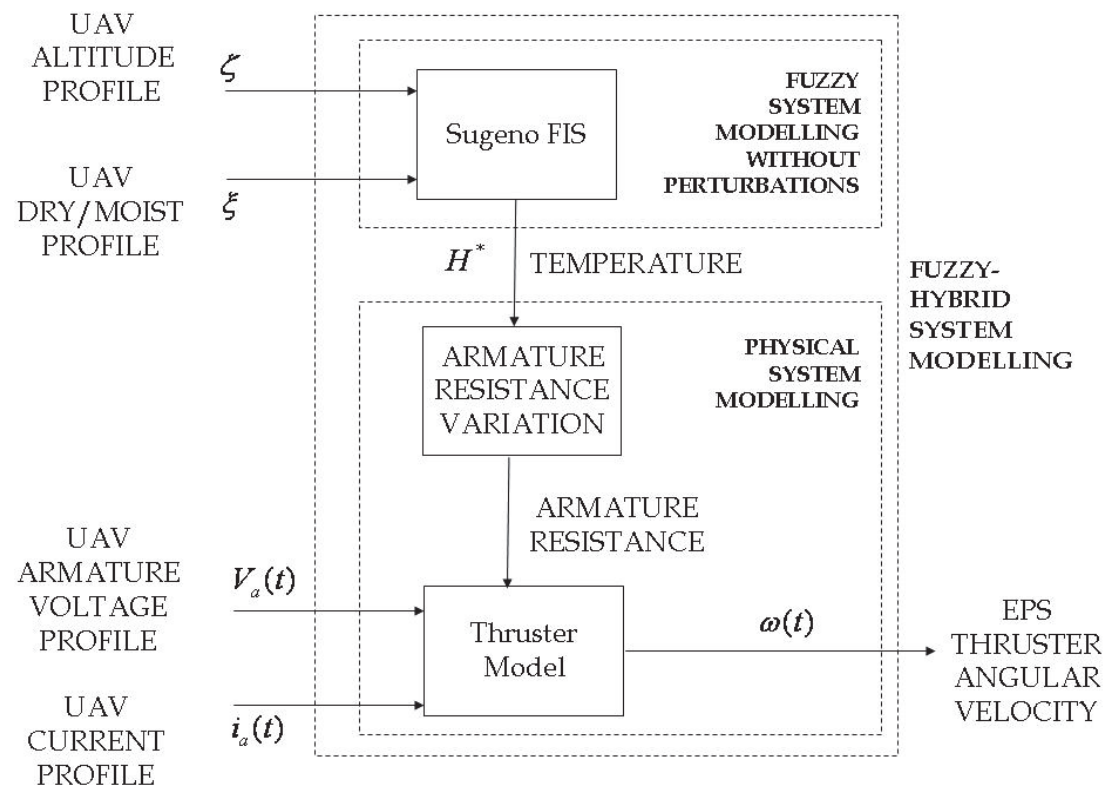

Fig. 7. UAV EPS Model at variable exogenous conditions with Sugeno (fuzzy-hybrid system) 


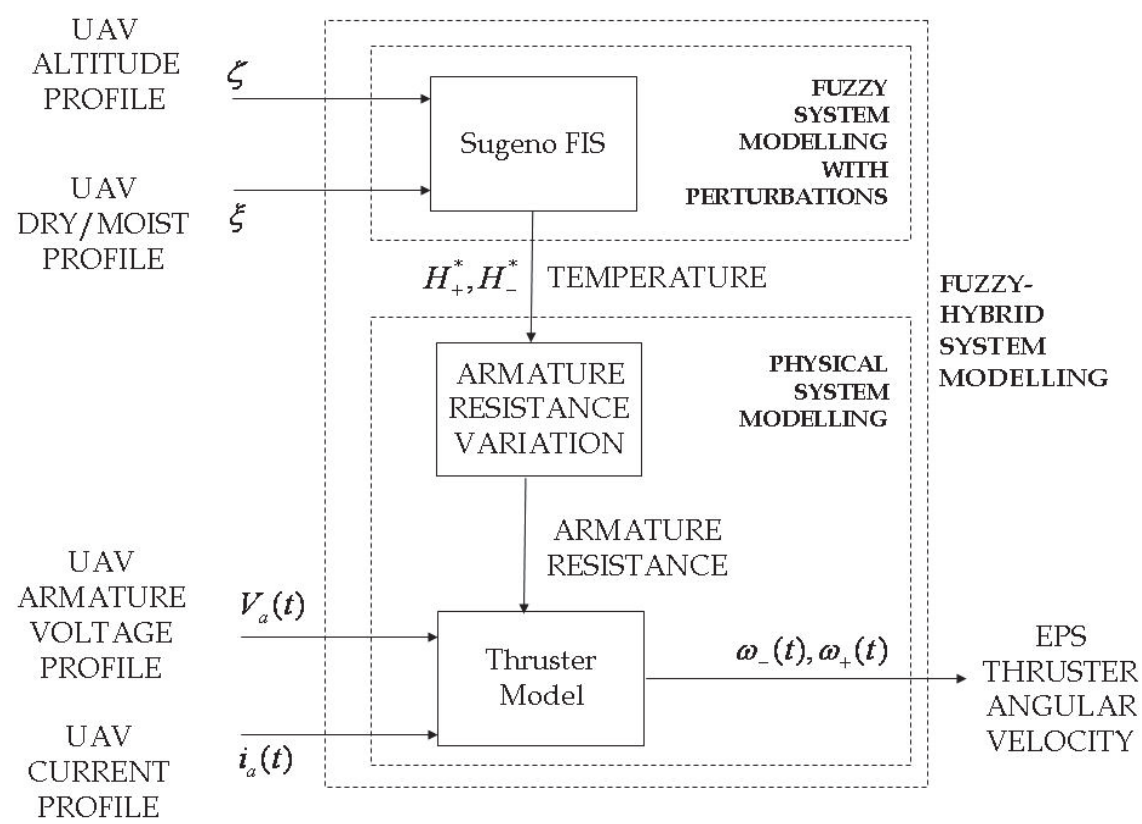

Fig. 8. UAV EPS Model at variable exogenous conditions with Sugeno (fuzzy-hybrid system) and Sugeno parameter perturbations.

The inputs of Figure 5 are shown next in Figure 9 (top, centre graphs) while the resulting thruster's input electrical power is also shown (lower graph). The quasi-static approach shows that the armature input electrical power does vary in order to balance the UAV flight requirements for altitude and overcome the atmospheric air moisture conditions.

Clearly, Figure 10, shows a realisable UAV test scenario. Initially the UAV starts at ground (sea level) and gradually gains altitude with a realisable climb rate. During its mission the UAV remains at a fixed altitude and then gains altitude again reaching before its $6 \mathrm{~km}$ requirement, where it remains for a given time $(25 \mathrm{~min})$ until it starts to descend back to sea level.

Meanwhile, the air moisture varies between two fuzzy logic extremes of " 1 " and " 0.5 " each representing a different condition, "dry air" and "saturated moist air" respectively. The moist air affects the temperature variation as the UAV altitude varies and hence was modelled utilising the Sugeno FIS topology.

Based on the chapter hypothesis, the armature resistance will affect the propeller shaft angular velocity for given conditions. Therefore, the next step is to observe the armature resistance during the UAV mission and compare this to the nominal (sea level) conditions. Figure 11, successfully demonstrates the nominal "blue line" armature resistance at sea level and the variable resistance due to the altitude and air moisture conditions.

In Figure 11, the dotted upper and lower lines demonstrate the injected $\pm 10 \%$ perturbation in the Sugeno consequent. Both the effects of altitude, air moisture and the sensor SIEB type of perturbations affect the thruster's armature resistance and therefore it is expected to observe this variation to cascade also to the thruster's variables such as the propeller shaft angular velocity. 
Figure 12 , shows more clearly the injected $\pm 10 \%$ perturbations in the Sugeno consequent and the effect of these. Typically, the boundaries (upper and lower) indicate the line for instantaneous measurements where the sensor measurement is used rather than the exact value of the sensor.
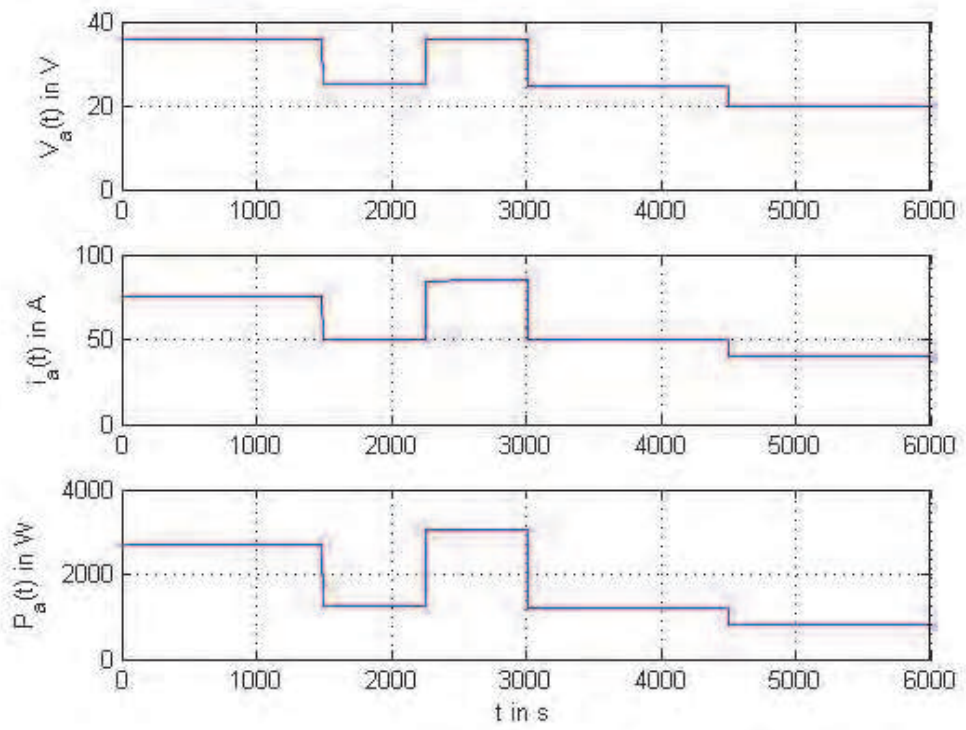

Fig. 9. UAV thruster armature voltage, current and input electrical power.
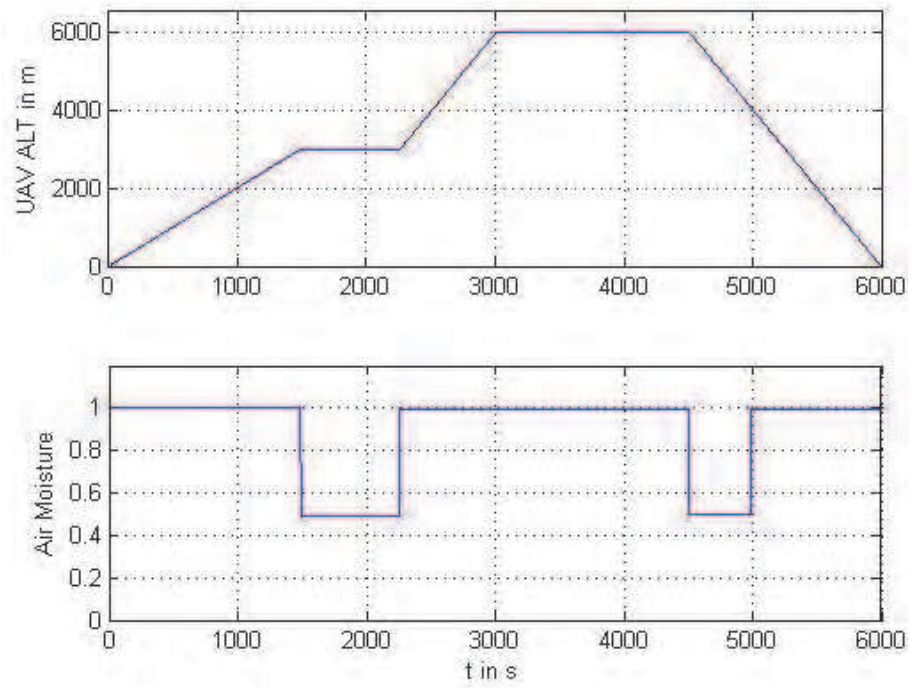

Fig. 10. UAV operational scenario, indicating altitude and air moisture conditions. 


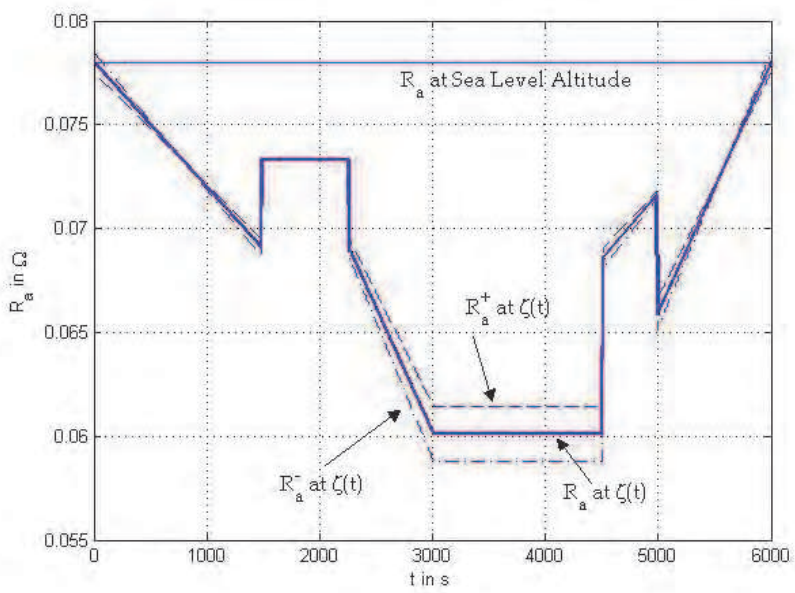

Fig. 11. Thruster armature resistance for nominal conditions (blue) and altitude based conditions (red).

Normally, UAV propulsion pack designs have a limited maximum rated electrical power which is available for use, including the propeller power requirements and thruster's power losses. Figure 12, shows the armature resistance related copper losses for the given UAV test scenario. Clearly, the power copper losses relating to the nominal (sea level) when compared to the variable altitude and air moisture conditions result in different losses. In particular the variable altitude scenario power losses are less than the sea level equivalent, hence resulting in a gain in net power available for thrust for the same power pack.
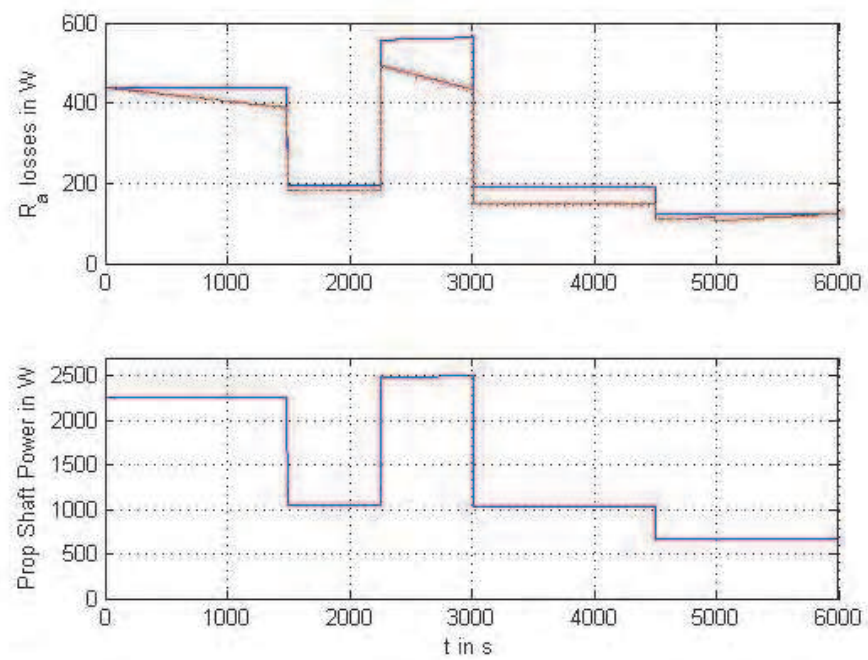

Fig. 12. Nominal (blue) and altitude based copper losses and propeller shaft powers. 
Figure 12, (lower graph), shows the propeller shaft available power for the test scenario shown earlier. During time intervals $(0,1500) \mathrm{s}$ and $(2200,3000) \mathrm{s}$ the UAV requires its maximum rated power in order to climb to the desired altitudes of $3000 \mathrm{~m}$ and $6000 \mathrm{~m}$.
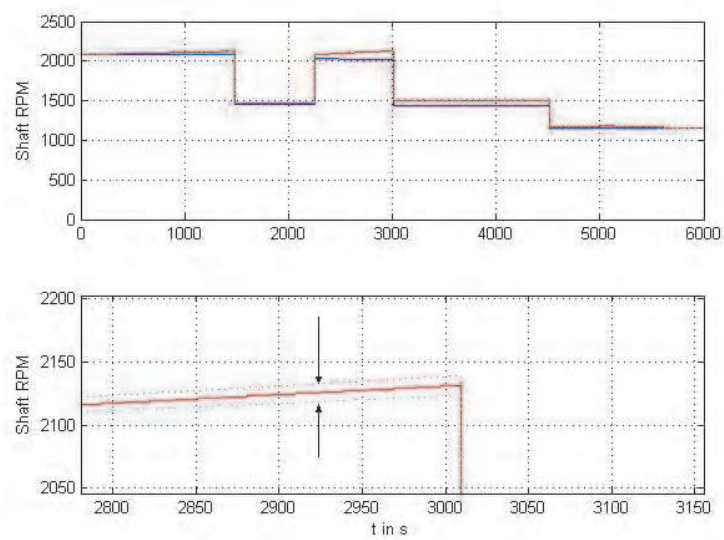

Fig. 13. Geared shaft RPM for nominal (blue) and altitude based (red), second graph showing the percent variation in the shaft RPM.

Figure 13, shows (top graph) the propeller geared shaft RPM for the nominal (in blue) and the altitude varied angular velocity (in red). As expected because the power pack has a maximum rated power capability and the armature resistance losses reduce, the propeller shaft mechanical power increases for the same rated input power. Hence, while the propeller loading remains as shown in the previous profiles the angular velocity at the propeller shaft is expected to increase as shown from the analysis.

Figure 13, also shows a zoomed version (lower graph) clearly showing the implications of the added phenomenon of speed changing due to an example injected $\pm 10 \%$ perturbations in the Sugeno sensor. It appears that this specific injected perturbation does not cause a substantial change compared to the altitude based angular velocities.

Figure 14. shows the armature resistance percentage error when compared to the sea level conditions. Clearly the expected error (top graph) exceeds $20 \%$ from nominal, therefore demonstrating the importance of the Sugeno fuzzy inference modelling within the context of the fuzzy-hybrid modelling process. The armature resistance percentage error for both the upper and lower boundaries (centre graph), are approximately $2.5 \%$ for the upper/lower boundary or 5\% for both boundaries. This indicates that the Sugeno perturbation based on SIEB-type errors can indeed affect the model behaviour. The (last graph), shows the SIEB errors with reference to the sea level equivalent. These are expected to be high and exceeding $20 \%$ due to the inclusion of the fuzzy-hybrid model which includes the altitude/moisture and perturbation effects.

Figure 15 shows the thruster's angular velocity error comparing the sea level and altitude based models. Clearly the error (top graph) is nearly $5 \%$ and variant throughout the UAV flight scenario. The centre graph shows the thruster's upper and lower injected $\pm 10 \%$ perturbations in the Sugeno FIS and compared to the non-perturbation model. The error resulting from this test run is less than $1 \%$, thus shown some influence of the armature 
resistance variations cascading and affecting the propeller shaft angular velocity. However, (last graph), when the perturbation model is compared to the sea level model the error increased by approximately 10 times reaching a percentage error of up to $6 \%$.
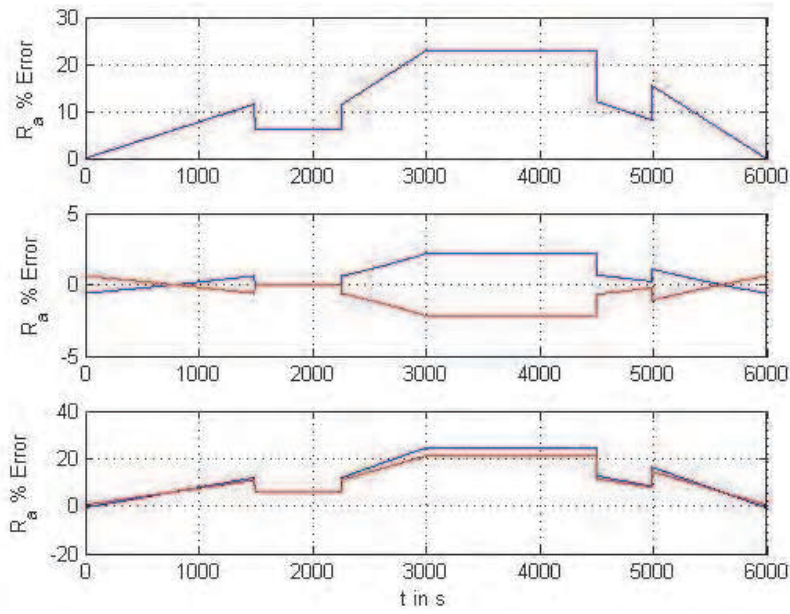

Fig. 14. Altitude-based armature resistance error with respect to the nominal (top graph); altitude-based armature resistance error wrt $\pm 10 \%$ FIS Consequent perturbation (Centre graph); the lower graph is showing the error due to $\pm 10 \%$ FIS Consequent perturbation wrt the nominal armature resistance.
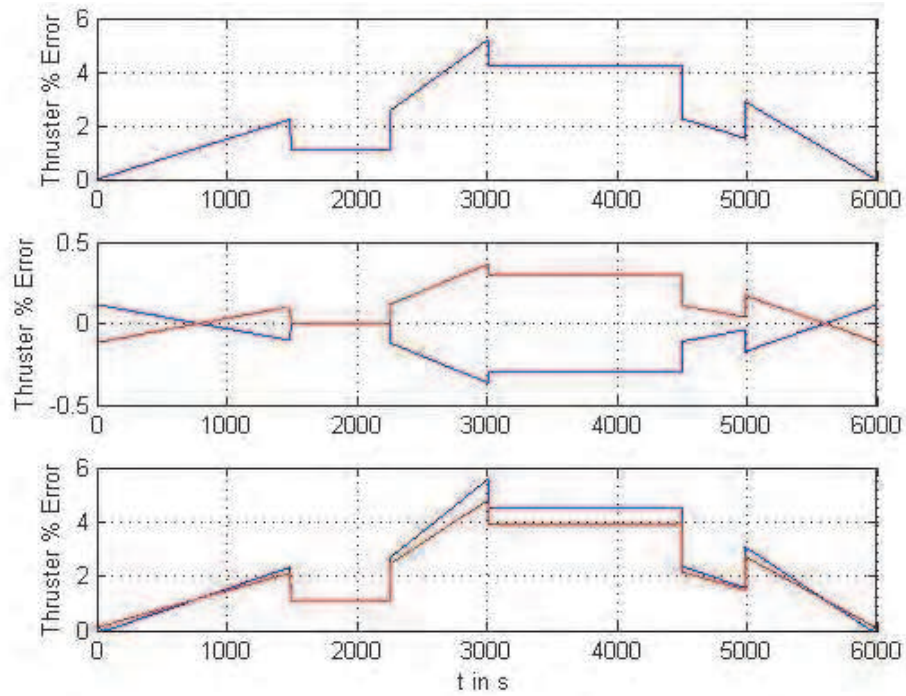

Fig. 15. Altitude-based shaft angular velocity error with respect to the nominal (top graph); altitude-based angular velocity error wrt $\pm 10 \%$ FIS Consequent perturbation (Centre graph); the lower graph is showing the error due to $\pm 10 \%$ FIS Consequent perturbation wrt the nominal angular velocity. 


\section{Conclusion}

In this chapter we have learned how to incorporate sensor perturbations via the Sugeno fuzzy logic inference for electrical thruster systems which are propelling a class of electrically-powered unmanned aerial vehicles. Therefore, design considerations have included the UAV altitude variation and atmospheric moisture via the fuzzy logic Sugeno design framework.

Furthermore the necessity of the fuzzy-hybrid modelling topology became apparent for the electrical thruster system. While the thruster was modelled utilising an ordinary differential equation form, the additional UAV operational conditions such as altitude and atmospheric moisture required the inclusion of the Sugeno-based fuzzy inference system thus amalgamating the two topologies into a single fuzzy-hybrid topology.

\section{Nomenclature}

\begin{tabular}{|c|c|}
\hline & Nomenclature (Units are in SI) \\
\hline$n$ & Rule number \\
\hline$n_{\max }$ & Maximum number of rules \\
\hline$j$ & Number of Sensors \\
\hline$j_{\max }$ & Maximum number of sensors \\
\hline$z_{j}$ & j-th sensor variable \\
\hline$Z_{n}^{j}$ & Membership function for the \\
\hline$h_{n}$ & $\mathrm{n}$-th rule function \\
\hline$f_{n}\left(z_{1}, z_{2}, z_{3}\right)$ & Linear polynomial in terms of $\mathrm{z}_{1}, \mathrm{z}_{2}, \ldots, \mathrm{z}_{\mathrm{j}}$ \\
\hline$c_{j}$ & Centre for Gaussian type membership function for the $\mathrm{j}$-th sensor \\
\hline$d_{j}$ & Dispersion for Gaussian type membership function for the j-sensor \\
\hline$\eta$ & Horizontal shift operator \\
\hline$b_{n}$ & Rule consequent offset \\
\hline$\varsigma_{j n}$ & n-th rule $j$-th sensor polynomial coefficients \\
\hline$h^{*}$ & Sugeno FIS output at time $\tau$. \\
\hline$\gamma_{n}$ & $\mathrm{n}$-th rule rule firing \\
\hline$\varepsilon_{j}$ & j-th sensor error \\
\hline $\bar{\varepsilon}_{j}$ & $\mathrm{j}$-th sensor error upper boundary \\
\hline$\underline{\varepsilon}_{j}$ & $j$-th sensor error lower boundary \\
\hline$V_{a}(t)$ & PMDC Armature thruster voltage \\
\hline$\omega(t)$ & Thruster angular velocity \\
\hline$i_{a}(t)$ & Thruster Armature Current \\
\hline$K_{a}$ & Thruster back emf constant \\
\hline$R_{a}$ & Thruster armature resistance \\
\hline$R_{a}^{+}$ & $\begin{array}{l}\text { Sugeno upper bound for armature resistance for different } \\
\text { altitudes }\end{array}$ \\
\hline
\end{tabular}




$\begin{array}{ll}R_{a}^{-} & \text {Sugeno lower bound for armature resistance for different altitudes } \\ L & \text { Thruster inductance } \\ E_{a} & \text { Thruster equivalent back emf voltage } \\ \theta(t) & \text { Shaft angle } \\ K_{T} & \text { Thruster torque constant } \\ T_{m} & \text { Thruster produced torque } \\ J_{a} & \text { Thruster armature inertia } \\ J_{L} & \text { Load inertia } \\ B_{a} & \text { Thruster armature viscous angular damping } \\ B_{L} & \text { Load viscous angular damping } \\ N_{1} & \text { Thruster side gear teeth } \\ N_{2} & \text { Load side gear teeth } \\ P_{m} & \text { Thruster mechanical power } \\ \rho & \text { Constant in } W \\ R_{r e f} & \text { Reference resistance for thrusters armature at temperature } T_{r e f} \\ a_{c} & \text { Coefficient of thermal expansion for copper } \\ \zeta & \text { UAV Altitude in m } \\ \xi & \text { Air moisture condition } \\ T & \text { Temperature at altitude } \zeta \\ T_{r e f} & \text { Reference temperature }\end{array}$

\section{References}

Kladis, G.P.; Economou, J.T.; Knowles, K.; Lauber, J. \& Guerra T.M. (2010). Energy conservation based fuzzy tracking for unmanned aerial vehicle missions under a priori known wind information, Journal of Engineering Applications of Artificial Intelligence, Vol. 24, Issue 2, pp. 278-294.

Karunarathne, L.; Economou J.T. \& Knowles, K. (2007). Adaptive neuro fuzzy inference system-based intelligent power management strategies for fuel cell/battery driven unmanned aerial vehicles, Journal of Aerospace Engineering, Vol/ 224, No. G1, pp $77-88$.

Sugeno, M. (1999). On stability of fuzzy systems expressed by fuzzy rules with singleton consequents, IEEE Transactions on Fuzzy Systems, Vol. 7, Issue 2, pp 201-224.

Economou, J.T. \& Colyer, R.E. (2005). Fuzzy-hybrid modelling of an Ackerman steered electric vehicle, International Journal of Approximate Reasoning, Vol.41, No.3, pp. 343-368.

Ehsani, M.; Gao, Y.; Gay, S.E. \& Emadi, A., (2005). Modern Electric, Hybrid Electric, and Fuel Cell Vehicles, CRC Press, ISBN 084933154 4, USA.

Economou, J.T.; Tsourdos, A. \& White B.A. (2007). Fuzzy logic consequent perturbation analysis for electric vehicles, Journal of Automobile Engineering, Proceedings of the IMech E PART D., Vol. 221, No D7, pp 757-765. 
Miller, J.M., (2004). Propulsion Systems for Hybrid Vehicles, IEE Power \& Energy Series 45, ISBN 086341336 6, UK. 


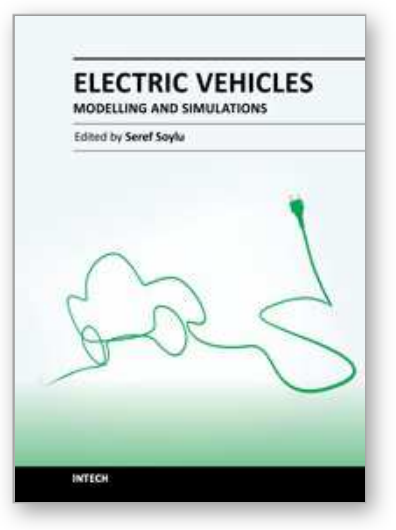

\author{
Electric Vehicles - Modelling and Simulations \\ Edited by Dr. Seref Soylu
}

ISBN 978-953-307-477-1

Hard cover, 466 pages

Publisher InTech

Published online 12, September, 2011

Published in print edition September, 2011

In this book, modeling and simulation of electric vehicles and their components have been emphasized chapter by chapter with valuable contribution of many researchers who work on both technical and regulatory sides of the field. Mathematical models for electrical vehicles and their components were introduced and merged together to make this book a guide for industry, academia and policy makers.

\title{
How to reference
}

In order to correctly reference this scholarly work, feel free to copy and paste the following:

John T. Economou and Kevin Knowles (2011). Sugeno Inference Perturbation Analysis for Electric Aerial Vehicles, Electric Vehicles - Modelling and Simulations, Dr. Seref Soylu (Ed.), ISBN: 978-953-307-477-1, InTech, Available from: http://www.intechopen.com/books/electric-vehicles-modelling-and-simulations/sugenoinference-perturbation-analysis-for-electric-aerial-vehicles

\section{INTECH}

open science | open minds

\section{InTech Europe}

University Campus STeP Ri

Slavka Krautzeka 83/A

51000 Rijeka, Croatia

Phone: +385 (51) 770447

Fax: +385 (51) 686166

www.intechopen.com

\section{InTech China}

Unit 405, Office Block, Hotel Equatorial Shanghai

No.65, Yan An Road (West), Shanghai, 200040, China

中国上海市延安西路65号上海国际贵都大饭店办公楼 405 单元

Phone: +86-21-62489820

Fax: +86-21-62489821 
(C) 2011 The Author(s). Licensee IntechOpen. This chapter is distributed under the terms of the Creative Commons Attribution-NonCommercialShareAlike-3.0 License, which permits use, distribution and reproduction for non-commercial purposes, provided the original is properly cited and derivative works building on this content are distributed under the same license. 